Research report

\title{
Compensatory networks to counteract the effects of ageing on language
}

\author{
Rosa Manenti ${ }^{\mathrm{a}, *}$, Michela Brambilla ${ }^{\mathrm{a}, \mathrm{b}}$, Michela Petesi $^{\mathrm{a}}$, Carlo Miniussi ${ }^{\mathrm{a}, \mathrm{c}}$, Maria Cotelli $^{\mathrm{a}}$ \\ a IRCCS Centro San Giovanni di Dio Fatebenefratelli, Brescia, Italy \\ ${ }^{\mathrm{b}}$ Center for Cognitive Science, Department of Psychology, University of Turin, Turin, Italy \\ ${ }^{\mathrm{c}}$ Dept of Clinical and Experimental Sciences, National Institute of Neuroscience University of Brescia, Brescia, Italy
}

\section{H I G H L I G H T S}

- rTMS was used to influence the function of the prefrontal cortex.

- TMS effects on naming ability differed according to the subject's performance.

- Less prefrontal asymmetry is a strategy to counteract age-related naming decline.

\section{A R T I C L E I N F O}

\section{Article history:}

Received 21 February 2013

Received in revised form 8 April 2013

Accepted 11 April 2013

Available online 17 April 2013

\section{Keywords:}

TMS

Compensation

Naming

DLPFC

Elderly

\begin{abstract}
A B S T R A C T
Background: Word-retrieval difficulties are a common consequence of healthy ageing and are associated with a reduction in asymmetrical recruitment of the dorsolateral prefrontal cortex (DLPFC), although the significance of this reduction has not yet been clarified. Using repetitive transcranial magnetic stimulation (rTMS) it has been demonstrated that an asymmetrical involvement of the DLPFC during action naming in young subjects, whereas bilateral involvement was shown in elderly participants. By using rTMS during a naming task in a group of elderly subjects, the aim of the present work was to investigate whether the magnitude of DLPFC asymmetry (left-right rTMS effect) during action naming correlates with task performance, proving the presence of a compensation strategy in some but not all elderly participants. Methods: We aimed to test if there was a correlation between DLPFC asymmetry (left-right rTMS effect) and naming performance in a group of elderly subjects.

Results: The results show that rTMS affects action naming differently according to individual naming ability. In particular, the predominance of a left vs. right DLPFC effect was observed only in the lowperforming older adults, while an asymmetric reduction was selectively shown in the high-performing group. Interestingly, high-performing older adults also displayed better performances on a phonemic fluency test.

Conclusion: The present data suggest that successful ageing is linked to less prefrontal asymmetry, an efficient strategy for counteracting age-related declines in cognitive function.
\end{abstract}

(C) 2013 Elsevier B.V. All rights reserved.

\section{Introduction}

Ample evidence has illustrated the relationship between age-related changes in the brain and alterations in cognitive performance [1]. Studies at the neuronal level have demonstrated that during physiological ageing, dopaminergic decline as well as grey and white matter atrophy are both correlated with specific cognitive changes [2,3]. A recent review [4] concluded that the brain shrinks in volume and that the ventricular system expands in the ageing brain (with high heterogeneity). The volumetric reductions

\footnotetext{
* Corresponding author at: IRCCS Centro San Giovanni di Dio Fatebenefratelli, Via Pilastroni 4, 25125 Brescia, Italy. Tel.: +39 0303501593; fax: +39 0303533513.

E-mail address: rmanenti@fatebenefratelli.it (R. Manenti).
}

are likely related, to a minor extent, to neural loss because the shrinkage of neurons, reduction of synaptic spines and lower numbers of synapses probably account for the reduction in grey matter [4]. However, reductions in processing speed, memory, executive functions and language production have been shown during physiological ageing. Such reductions are mediated by neuroanatomical changes, with increased age often associated with lower performance on a wide variety of cognitive tasks [4,5]. Accordingly, it is clear that ageing is influenced by a large number of factors that vary from individual to individual, and, to date, it is unclear how differences in brain activity relate to cognitive performance in elderly participants [5,6]. Because the mean age of the population increases the number of people that will experience an age-related reduction in cognitive abilities will increase; despite this, although some individuals will show pronounced cognitive deficits, others will not. 
Understanding the basis of minor vs. major age-related cognitive decline is of great interest, and some investigations have assessed the predictive value of several factors for successful ageing $[7,8]$, and non-pharmacological interventions for cognitive difficulties in healthy older adults have gained much attention in recent years [9-11]. Relevant studies have suggested that cerebral plasticity and cognitive reserve play important roles in physiological ageing $[9,10,12,13]$.

One method used to investigate age-related modifications in cognition is neuroimaging, and neuroimaging data from some cognitive models on ageing have been acquired. A recent review highlights that the application of functional neuroimaging to healthy ageing participants suggests functional age-related changes during the execution of several cognitive tasks, despite the many discrepancies in this literature [14]. Alterations in hemispheric asymmetry have been reported in studies of physiological and pathological ageing [15-17]. Frontal asymmetry was significantly reduced during a cognitive task in healthy elderly participants as compared to young participants [16]. As one of the major cognitive declines in both healthy and pathological ageing is a reduction in memory performance, most of the neuroimaging studies on healthy ageing participants have investigated memory processes and suggest the presence of functional age-related reductions in the left prefrontal cortex (PFC) and temporo-occipital regions and increases in activity in the insular regions. Generally, these studies have shown a reduction in functional asymmetry in the prefrontal cortex [18] and have led to development of the hemispheric asymmetry reduction in older adults (HAROLD) model [16], subsequently confirmed by rTMS [16,19-24]. Additionally, over-activation of the PFC in older adults represents the starting point of the posterior-anterior shift in ageing (PASA) model, which implicates age-related increases in frontal activity and decreases in occipital lobe activity [25].

Language represents another example of a cognitive ability that dramatically changes during ageing. Evidence from both lesion and imaging studies suggests a central role for the left prefrontal, temporal and parietal areas during naming, although some differences exist for object (noun) vs. action (verb) naming [26-28]. A progressive reduction in lexical retrieval has been well-documented in older adults [29-31]. Unlike word-meaning retrieval, which appears to be preserved or even enhanced with ageing [32,33], the ability to retrieve the sound or phonology of words seems to decline in older adults. Moreover, the frequency of tip-of-the-tongue experiences significantly increases with ageing, and older adults report an inability to produce well-known words as one of the most annoying cognitive "symptoms" that they experience [34,35]. A few imaging studies have reported on a neural age-related change that correlates with the increase of this disturbance in healthy ageing. The results of these studies support a neural cause of word retrieval difficulties (tip-of-tongue effect) in physiological ageing, highlighting the effects of ageing on the activity of the insula [36] and in the white matter integrity of the posterior aspect of the superior longitudinal fasciculus [37].

Even if naming is an ability that shows adaptation during ageing, only a few studies have investigated how age-related changes in activity relate to performance during naming tasks in older adults.

Different hypotheses have been considered to explain the significance of these age-related changes. First, the compensation hypothesis [25,38-41] proposes that increased functional hemispheric symmetry in older adults is counteracted by age-related neurocognitive deficits. Second, the dedifferentiation hypothesis explains the reduced asymmetry as a difficulty in recruiting specialised neural networks [42,43]. Last, the default network theory postulates that the major components of the default mode network remain stable in healthy, older individuals, whereas the activity in a number of discrete cortical areas located in the prefrontal, temporal, and occipital regions changes over time [44]. There is a large degree of overlap among these hypotheses, but all of them at least partially underline the ability of cerebral tissue to change its structure and function continuously in response to environmental demands. To compare these hypotheses, several studies have investigated whether PFC functional symmetry is linked to a reduction or enhancement of memory performance, but the results are still inconclusive. Regarding naming tasks, only a few neuroimaging studies have shown age-induced increases in cortical activation in the brain areas typically associated with language processing (Broca's and Wernicke's areas) [45,46]. Interestingly, only one fMRI study has examined the role of functional age-related changes in naming abilities and found a positive correlation between BOLD responses, naming accuracy and response latencies in the frontal areas of older adults [46], verifying the presence of compensatory mechanisms in the frontal cortex that are invoked to maintain performance in healthy ageing. A recent review of the functional brain imaging correlates of successful cognitive ageing found frontal cortex responses in older, as compared to younger, individuals, independent of task [41].

However, functional neuroimaging data cannot prove the necessity of an area during a cognitive task because an activated area may simply be correlated with, rather than responsible for, task performance. In contrast, repetitive transcranial magnetic stimulation (rTMS) can induce a temporary impairment in performance only if the stimulated area is causally engaged in the task [47]. Based on this assumption, TMS has been used in many different cognitive domains to establish causality in brain-behaviour relationships and, specifically, to verify asymmetrical involvement of the PFC during selected tasks. Hemispheric specialisations of the dorsolateral PFC (DLPFC) during episodic memory have previously been demonstrated using rTMS in young subjects [19,21-23,48-52] and in elderly adults $[20,53]$. For an extensive review of this literature, please refer to Manenti et al. [24]. Regarding naming, an rTMS study of young participants showed a left predominance of the DLPFC during action naming [54]. A unique study that investigated the effects of physiological ageing on DLPFC asymmetry during action and object naming showed a selective bilateral facilitatory effect during action naming, namely a decrease in vocal reaction time, in a group of older adults [55].

The purpose of this study was to examine if the magnitude of DLPFC lateralisation (left-right rTMS effect) during action naming was correlated with task performance, proving the presence of a compensation strategy in some but not all elderly participants. In the present report, we re-analysed data acquired in our previous study [55] to thoroughly investigate this issue. Based on previous studies $[20,46,56]$, we predicted that DLPFC hemispherical symmetry during action naming would only be present in high-performing older adults. If identified, this result would prove that a reduction in DLPFC asymmetry in some but not all elderly participants represents a compensation strategy used to counteract age-related changes, resulting in better task performance.

\section{Materials and methods}

\subsection{Participants}

Prior to being enrolled in the experiment, participants were administered a standard health history questionnaire and completed a Mini Mental State Examination (MMSE) [57]. Potential participants were excluded if they reported a history of neurological disease, cardiovascular disease, psychiatric disorders or alcohol or other substance abuse. Individuals who reported subjective memory complaints or scored below 27 out of 30 on the MMSE were also excluded. In addition, a neuropsychological battery was applied, and a pathological score in at least one of the tests was a further exclusion criterion. Thirteen ( 4 male, 9 female) healthy older adults (age: $65-78$ years, mean $=70.2$ years, education: mean $=13.8$ years) participated in the rTMS experiment. All participants were native Italian speakers and had normal or corrected-to-normal vision. All participants were right-handed [58] and had no contraindications for rTMS [59]. This study was approved by the local ethics 
committee, and participants were informed about the possible risks of rTMS before informed consent was obtained.

The neuropsychological test battery included measures used to assess nonverbal reasoning (Raven's Coloured Progressive Matrices), language comprehension (Token Test), verbal fluency (phonemic and semantic), memory (Story Recall, ReyOsterrieth Complex Figure Recall, Digit Span, Spatial Span), visuo-spatial abilities (Rey-Osterrieth Complex Figure, Copy), attention and executive functions (TrailMaking Test A and B). All the tests were administered and scored according to standard procedures [60]. In addition, some subtests of the Battery for Analysis of Aphasic Deficits (BADA) were applied [61]. The results of the cognitive assessments are presented in Table 1.

\subsection{Stimuli}

The stimuli used in the action and object picture-naming tasks were provided by the Centre for Research in Language-International Picture Naming Project (CRLIPNP) [62]. We selected 84 items ( 42 actions and 42 objects) in a previous experiment [55], and none of the action stimuli included in this task were associated with the selected objects. The nouns and verbs corresponding to the sets of objects and actions were matched for target word frequency and length.

The items were divided into three blocks designed for the three stimulation conditions (left DLPFC, right DLPFC and sham). The frequencies and lengths of the target words were counterbalanced in each experimental block. The visual complexity and imageability of the pictures were also matched between blocks. Ten additional objects and actions were used as a practice block ( 5 actions and 5 objects). The presentation order was counterbalanced across subjects.

\subsection{Procedure}

\subsubsection{Naming task}

Subjects sat in front of a 17-in. monitor controlled by a personal computer running the Presentation software (www.neurobs.com). After a frame that indicated the category of the stimulus to the subject ("ACTION" or "OBJECT") was displayed, a $50 \mathrm{~ms}$ sound was presented upon the onset of a centrally located fixation cross. The fixation cross was present for $1000 \mathrm{~ms}$. After the fixation cross disappeared, the stimulus was presented and remained on the screen for $1000 \mathrm{~ms}$. A blank screen followed for anywhere from 4000 to $5000 \mathrm{~ms}$. The subject's task was to accurately name the stimuli appearing on the computer screen as quickly as possible. Verba responses were recorded and digitised at $44.1 \mathrm{kHz}$ using the GoldWave program (V. 5.12, www.goldwave.com). The responses were then analysed off-line to obtain accuracy and vocal reaction times (vRTs). For each stimulus, we calculated the mean vRT and the mean response accuracy percentage.

\subsubsection{Transcranial magnetic stimulation}

For left and right DLPFC stimulation, the Talairach coordinates of the cortical sites underlying the coil were estimated for each subject using a SofTaxic Evolution Navigator system (V. 2.0; www.emsmedical.net) and by identifying the stimulation site on the scalp above Brodmann area 8 (Talairach coordinates $X= \pm 35, Y=24, Z=48$, middle frontal). We used a $70 \mathrm{~mm}$ figure-eight coil placed above the target point to deliver rTMS for $500 \mathrm{~ms}$ from the onset of the visual stimulus at a frequency of $20 \mathrm{~Hz}$. The stimulation intensity used during the experiment was set at $90 \%$ of each subject's resting motor threshold. For the sham control condition, a 3-cmthick piece of plywood was applied to the coil so that no magnetic fields reached the cortex and the junction of the two coil wings was placed above the vertex ( $C Z$ in the EEG 10/20 international system). These parameters are in line with the safety recommendations for rTMS [59], and none of the subjects showed side effects of stimulation.

\subsection{Analysis of the link between hemispheric asymmetry and action naming performance}

We only analysed action naming task performance, following the results of our previous study that showed significant rTMS-induced effects exclusively during action naming [55]. Both the vRTs and accuracy were analysed. One index of interhemispheric asymmetry was computed at the single-subject level by examining the difference between the vRTs obtained in the right and left stimulation conditions.

Statistical analyses were performed using Statistica (version 10) software (www.statsoft.com). To test the compensation hypothesis, we calculated the $z$ points of the vRTs during action naming in the sham condition by dividing our participants into two groups: high-performing $(N=7)$ and low-performing $(N=6)$ Performance achieved in the sham condition was used to divide the participants into two groups because it represents the participants' baseline abilities. The distribution of $z$-points is characterised by a minimum of -1.1 and a maximum of +2.0 and the mean of this distribution (i.e., 0 ) is used as cut-off in order to distinguish between

\section{Table 1}

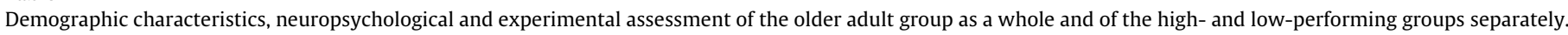
$p$ : $p$-value; ns: not significant.

\begin{tabular}{|c|c|c|c|c|c|}
\hline Demographic characteristics & All group $(N=13)$ & High-performing $(N=7)$ & Low-perf & $(n=6)$ & $p$ \\
\hline Age (years) & $70.2 \pm 4.0$ & $70.6 \pm 4.3$ & $69.8 \pm 3.5$ & & $\mathrm{~ns}$ \\
\hline Education (years) & $13.8 \pm 4.9$ & $13.9 \pm 4.8$ & $13.8 \pm 4.9$ & & ns \\
\hline Edinburgh Handedness Inventory & $+99.6 \pm 1.4$ & $+100 \pm 0.0$ & $+99.2 \pm 2$ & & ns \\
\hline Gender (female/male) & $9 / 4$ & $4 / 3$ & $5 / 1$ & & $\mathrm{~ns}$ \\
\hline Neuropsychological assessment & All group $(N=13)$ & High-performing $(N=7)$ & Low-performing $(N=6)$ & Cut-off & $p$ \\
\hline \multicolumn{6}{|l|}{ Screening for dementia } \\
\hline MMSE & $29.5 \pm 0.8$ & $29.6 \pm 0.8$ & $29.5 \pm 0.8$ & $\geq 24$ & ns \\
\hline \multicolumn{6}{|l|}{ Non-verbal reasoning } \\
\hline Raven-Coloured Progressive Matrices & $30.9 \pm 2.3$ & $31.7 \pm 2.8$ & $30.0 \pm 1.4$ & $>17.5$ & ns \\
\hline \multicolumn{6}{|l|}{ Memory } \\
\hline Story Recall & $15.2 \pm 2.0$ & $14.6 \pm 2.0$ & $15.8 \pm 1.8$ & $>7.5$ & ns \\
\hline Rey-Osterrieth Complex Figure, Recall & $14.6 \pm 4.4$ & $15.9 \pm 4.5$ & $13.2 \pm 4.1$ & $>9.46$ & ns \\
\hline Digit Span & $5.7 \pm 0.9$ & $5.4 \pm 1.0$ & $6.0 \pm 0.6$ & $>3.5$ & ns \\
\hline Spatial Span & $4.9 \pm 0.5$ & $4.7 \pm 0.5$ & $5.2 \pm 0.4$ & $>3.5$ & ns \\
\hline \multicolumn{6}{|l|}{ Praxia } \\
\hline Rey-Osterrieth Complex Figure, Copy & $33.1 \pm 1.7$ & $33.1 \pm 2.0$ & $33.1 \pm 1.5$ & $>28.87$ & ns \\
\hline \multicolumn{6}{|l|}{ Executive function } \\
\hline Trail-Making Test A (s) & $40.2 \pm 9.1$ & $43.3 \pm 8.2$ & $36.5 \pm 9.3$ & $<93$ & ns \\
\hline Trail-Making Test B (s) & $117.1 \pm 41.6$ & $128.7 \pm 44.1$ & $103.5 \pm 37.6$ & $<282$ & ns \\
\hline \multicolumn{6}{|l|}{ Language } \\
\hline Token Test & $33.6 \pm 1.3$ & $33.7 \pm 1.5$ & $33.5 \pm 1.0$ & $>26.25$ & ns \\
\hline Fluency, Phonemic & $39.6 \pm 8.2$ & $43.6 \pm 7.3$ & $35.0 \pm 7.0$ & $>16$ & 0.05 \\
\hline Fluency, Semantic & $45.3 \pm 7.6$ & $45.0 \pm 10.0$ & $45.7 \pm 4.1$ & $>24$ & ns \\
\hline Object Oral Comprehension (BADA) & $99.6 \pm 1.4$ & $100.0 \pm 0.0$ & $99.2 \pm 2.0$ & & ns \\
\hline Action Oral Comprehension (BADA) & $99.6 \pm 1.4$ & $100.0 \pm 0.0$ & $99.2 \pm 2.0$ & & ns \\
\hline Object Written Comprehension (BADA) & $99.8 \pm 0.7$ & $100.0 \pm 0.0$ & $99.6 \pm 1.0$ & & ns \\
\hline Action Written Comprehension (BADA) & $99.6 \pm 1.4$ & $99.3 \pm 1.9$ & $100.0 \pm 0.0$ & & ns \\
\hline Oral Object Naming (BADA) & $97.2 \pm 2.3$ & $97.6 \pm 2.5$ & $96.7 \pm 2.1$ & & ns \\
\hline Oral Action Naming (BADA) & $96.4 \pm 2.9$ & $95.9 \pm 2.5$ & $97.0 \pm 3.5$ & & ns \\
\hline \multicolumn{6}{|c|}{ Experimental assessment during Sham condition } \\
\hline \multicolumn{6}{|c|}{ Action naming } \\
\hline Correctness, $\%$ & $87.4 \pm 13.7$ & $88.4 \pm 10.0$ & $85.7 \pm 18.1$ & & ns \\
\hline Vocal reaction times, ms & $1077.5 \pm 129.3$ & $978.9 \pm 35.1$ & $1192.4 \pm 95.9$ & & 0.0001 \\
\hline
\end{tabular}

Raw scores are reported. Cut-off scores referred to Italian normative data. Bold scores highlight significant differences between high- and low-performing groups. 
the two groups. We used t-tests comparisons in order to compare performances achieved in the two elderly groups. Accordingly, we determined if any standard neuropsychological test was able to differentiate between participants with better performance and participants with reduced accuracy (see Table 1). Importantly, we investigated differences in the two groups using our index of interhemispheric asymmetry.

Considering the two groups, we compared the index of interhemispheric asymmetry of the two older participant groups with the mean left-right asymmetry (mean $=-120 \mathrm{~ms}$ ) of young individuals [54]. A similar analysis was conducted to compare the vRTs during action naming in the high- and low-performing groups of older adults (sham condition as baseline) with the mean vRTs during action naming in young participants (mean $=911 \mathrm{~ms}$ ).

Finally, the index of interhemispheric asymmetry was regressed against the vRTs and accuracy in the sham condition (i.e., individual abilities). A linear algorithm was applied to investigate whether greater asymmetry corresponded to better or worse performance on the action naming task.

The level of significance was set at $p<0.05$.

\section{Results}

\subsection{High- and low-performing elderly groups}

The high-performing $(N=7)$ and low-performing $(N=6)$ groups did not differ in age or education $(p>0.05)$. The two groups did not differ in action naming accuracy, although the accuracy was slightly lower in the low-performing group. Independent sample $t$-tests on the results of the neuropsychological tests highlight better scores in phonemic verbal fluency in the high-performing group $(t=2.17, p=0.05)$. The index of interhemispheric asymmetry was different between the two groups, with a significantly lower score (higher asymmetry) for the low-performing group $(t=5.04$, $p=0.002)$. Fig. 1 A shows a graphical representation of mean score at phonemic verbal fluency test ( $y$-axis) plotted separately for the two groups (mean index of interhemispheric asymmetry on the $x$-axis). None of the other tests showed significance.

\subsection{High- and low-performing elderly groups vs. young group}

A comparison of the two older groups with the young group showed similar DLPFC asymmetry in the low-performing group $(p>0.05)$ and a significant difference in asymmetry in the highperforming group $(t=5.04, p=0.002)$ as compared to the young group [54]. Furthermore, this analysis showed faster vRTs in the young group as compared to both the high- $(t=5.1, p=0.002)$ and low- $(t=7.2, p=0.0008)$ performing older adults, even though the high-performing older group showed shortened vRTs as compared to the low-performing older group $(t=-5.5, p=0.0001)$. These results suggest that the high-performing older adults significantly reduced DLPFC functional asymmetry to obtain better performance. Fig. 1B shows a graphical representation of mean vRTs during the experimental naming task ( $y$-axis) plotted separately for the two elderly groups and for the young group [54] (mean index of interhemispheric asymmetry on the $x$-axis).

\subsection{Correlations}

A significant negative correlation $(R=0.68, t=3.07, p=0.01)$ between the index of interhemispheric asymmetry and the vRTs in the sham condition suggests that the slower participants were characterised by greater involvement of the left hemisphere. Accordingly, a significant positive correlation $(R=0.54, t=2.17$, $p=0.05$ ) between the index of interhemispheric asymmetry and accuracy in the sham condition indicates that the participants that achieved better performance showed reduced involvement of the left hemisphere. Fig. 2 presents a detailed representation of these results (vRTs and accuracy on $y$-axis and the index of interhemispheric asymmetry on $x$-axis).
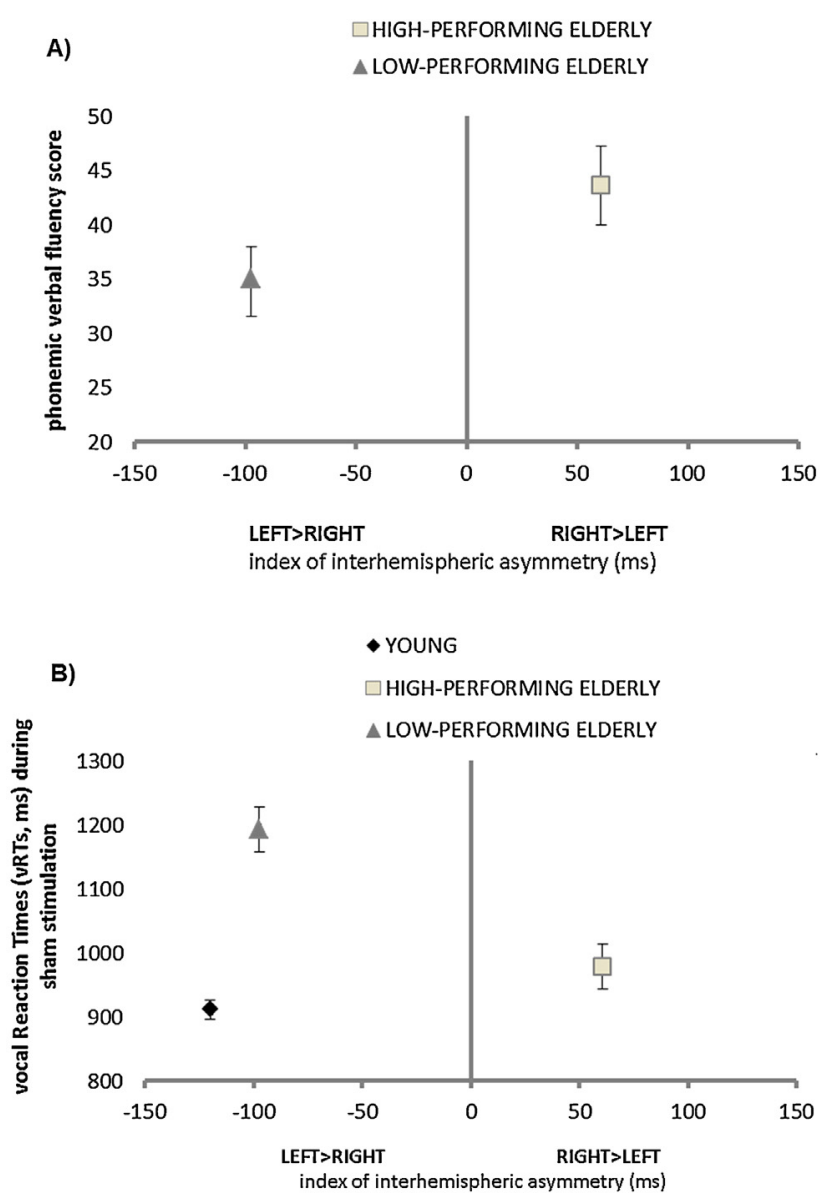

Fig. 1. (A) Mean phonemic verbal fluency score in older participants with high $(N=7)$ or low $(N=6)$ action naming performance. Interestingly, high-performing elderly participants showed less left hemisphere involvement and better phonemic verbal fluency scores, whereas the results from the low-performing elderly participants showed the opposite pattern. (B) Mean vocal reaction times (vRTs) in young ([54]; $N=9)$ and older participants with high $(N=7)$ or low $(N=6)$ action naming performance. Higher involvement of the left hemisphere (i.e., negative differences between vRTs in the left and right rTMS conditions) is evident in young participants and in older participants with low performances, whereas older participants with high performances show right or bilateral involvement of the DLPFC. Interestingly, the vRTs of the high-performing elderly participants are much more similar to those of young participants, but the asymmetry in the high-performing elderly participants is significantly different than that of young individuals. In order to obtain better performance, functional reorganisation is needed. Low-performing older adults show the same DLPFC asymmetry as young individuals but with significantly higher vRTs.

\section{Discussion}

The primary goal of the present study was to examine if the asymmetric reduction shown in older adults, as compared to young individuals, could be a compensation strategy used to counteract ageing. Accordingly, we investigated the influence of left and right DLPFC rTMS on performance during action naming.

We applied rTMS during action naming in healthy, high- or lowperforming, older participants and found that greater differences between left and right DLPFC involvement during action naming resulted in higher accuracy and faster responses in this task. These results suggest selective asymmetrical recruitment of the DLPFC (left > right) during action naming in low-performing older adults, as previously described in young individuals, whereas highperforming older adults did not show predominance (left = right). Low-performing older adults recruited a similar network of brain regions as the young adults but used them inefficiently. These findings have to be discussed in terms of functional changes in 

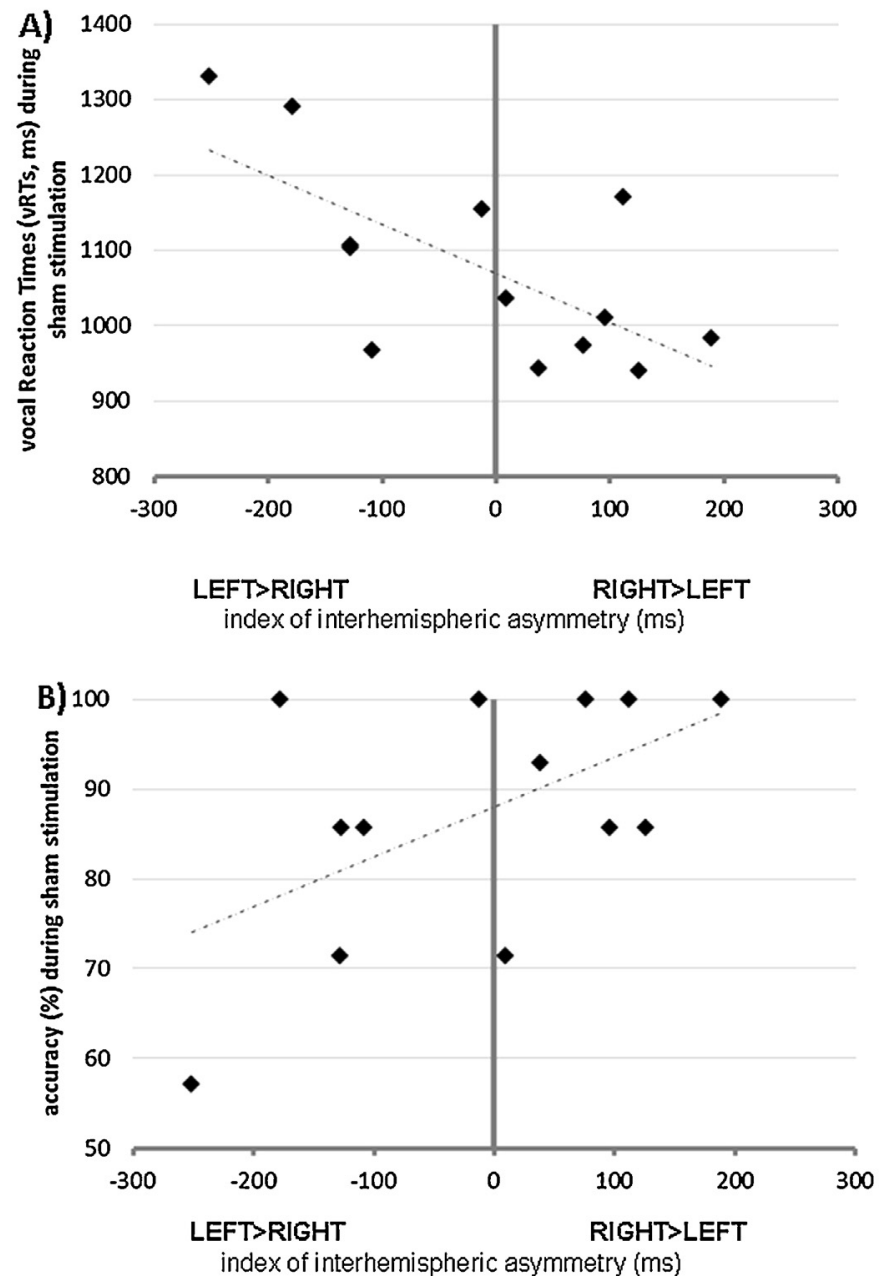

Fig. 2. Correlations between the index of interhemispheric asymmetry (verbal reaction times during left DLPFC stimulation minus verbal reaction times during right DLPFC stimulation) in action naming and the verbal reaction times (A) or accuracy (B) during sham conditions.

successful ageing [41]. In a recent review of the functional brain imaging correlates of successful cognitive ageing, Eyler et al. [41] concluded that augmented brain responses, particularly in the frontal cortex, serve a compensatory role in older adults, but in some cases, extraneous activation might be detrimental (i.e., in the posterior regions). Our results are in line with those of Eyler et al. [41], as frontal cortex functional recruitment was selectively modified during successful ageing (i.e., in high-performing older adults).

Our results seem to be in agreement with the compensation hypothesis, which has been investigated in the memory domain [63], because DLPFC recruitment was modified only in the high-performing participants, as compared to young participants. However, this result does not fit the dedifferentiation hypothesis $[42,43]$. If reduced lateralisation is merely another example of the deleterious effects of ageing on the brain, then it should have been found in the low-performing elderly group of adults because they displayed more pronounced age-related cognitive deficits; however, that was not the case. On the contrary, reduced lateralisation was found in the group of high-performing participants, suggesting that this feature might be considered as a beneficial change. Moreover, the high-performing participants achieved higher scores in phonemic fluency, underling better word finding abilities in these participants.
Our group found similar results in a previous study that also used brain stimulation during an episodic memory task [20]. We verified that during the encoding of word pairs, only high-performing older adults showed bilateral DLPFC involvement, whereas a left predominance, as observed in young individuals, was present in the low-performing participants. As reported for naming in the present work, functional modification of the DLPFC was positively correlated with better memory performance, suggesting that these changes represent a compensatory strategy in successful ageing. Importantly, agreement in the results regarding the different cognitive abilities strengthens the compensation hypothesis. We acknowledge that the relatively small number of elderly participants included in our study represent a limitation. However, the finding of this study could pave the way to further studies with a larger sample.

Although further studies are needed to confirm present results and clarify the basis of these effects, the possibility to better define the presence of ageing strategies responsible for impaired or maintained cognitive ability it is also important for designing rehabilitation protocols in a patient with language disabilities [64-69]

Methods to reduce or magnify inter-individual differences in performance, and the integration of these mechanisms with functional age-related plasticity, may have practical and societal implications. A greater understanding of how these patterns of compensatory strategies develop and help to maintain cognitive function in ageing is an important goal for future research.

\section{References}

[1] Salthouse TA. Effects of age on time-dependent cognitive change. Psychological Science 2011;22:682-8.

[2] Kramer JH, Mungas D, Reed BR, Wetzel ME, Burnett MM, Miller BL, et al. Longitudinal MRI and cognitive change in healthy elderly. Neuropsychology 2007;21:412-8.

[3] Sullivan EV, Rohlfing T, Pfefferbaum A. Longitudinal study of callosal microstructure in the normal adult aging brain using quantitative DTI fiber tracking. Developmental Neuropsychology 2010;35:233-56.

[4] Fjell AM, Walhovd KB. Structural brain changes in aging: courses, causes and cognitive consequences. Reviews in the Neurosciences 2010;21:187-221.

[5] Reuter-Lorenz PA, Park DC. Human neuroscience and the aging mind: a new look at old problems. Journals of Gerontology Series B, Psychological Sciences and Social Sciences 2010;65:405-15.

[6] Grady C. The cognitive neuroscience of ageing. Nature Reviews Neuroscience 2012;13:491-505.

[7] Formiga F, Ferrer A, Alburquerque J, Fernandez-Quevedo M, Royo C, Pujol R, et al. The challenge of maintaining successful aging at 87 years old: the octabaix study two-year follow-up. Rejuvenation Research 2012;15:584-9.

[8] Howieson DB, Camicioli R, Quinn J, Silbert LC, Care B, Moore MM, et al. Natural history of cognitive decline in the old old. Neurology 2003;60:1489-94.

[9] Ball K, Berch DB, Helmers KF, Jobe JB, Leveck MD, Marsiske M, et al. Effects of cognitive training interventions with older adults: a randomized controlled trial. Journal of American Medical Association 2002;288:2271-81.

[10] Acevedo A, Loewenstein DA. Nonpharmacological cognitive interventions in aging and dementia. Journal of Geriatric Psychiatry and Neurology 2007;20:239-49.

[11] Willis SL, Tennstedt SL, Marsiske M, Ball K, Elias J, Koepke KM, et al. Long-term effects of cognitive training on everyday functional outcomes in older adults. Journal of American Medical Association 2006;296:2805-14.

[12] Stern Y. Cognitive reserve and Alzheimer disease. Alzheimer Disease and Associated Disorders 2006;20:S69-74.

[13] Valenzuela MJ, Sachdev P. Assessment of complex mental activity across the lifespan: development of the Lifetime of Experiences Questionnaire (LEQ). Psychological Medicine 2007;37:1015-25.

[14] Guidotti Breting LM, Tuminello ER, Duke Han S. Functional neuroimaging studies in normal aging. Current Topics in Behavioral Neurosciences 2012;10:91-111.

[15] Cherbuin N, Reglade-Meslin C, Kumar R, Sachdev P, Anstey KJ. Mild cognitive disorders are associated with different patterns of brain asymmetry than normal aging: the PATH through life study. Front Psychiatry 2010;1:11.

[16] Cabeza R. Hemispheric asymmetry reduction in older adults: the HAROLD model. Psychology and Aging 2002;17:85-100.

[17] Long X, Zhang L, Liao W, Jiang C, Qiu B. The Alzheimer's Disease Neuroimaging. I. Distinct laterality alterations distinguish mild cognitive impairment and Alzheimer's disease from healthy aging: statistical parametric mapping with high resolution MRI. Human Brain Mapping 2012, http://dx.doi.org/10.1002/hbm.22157, in press. 
[18] Cabeza R, Grady CL, Nyberg L, McIntosh AR, Tulving E, Kapur S, et al. Age-related differences in neural activity during memory encoding and retrieval: a positron emission tomography study. Journal of Neuroscience 1997;17:391-400.

[19] Floel A, Poeppel D, Buffalo EA, Braun A, Wu CW, Seo HJ, et al. Prefrontal cortex asymmetry for memory encoding of words and abstract shapes. Cerebral Cortex 2004;14:404-9.

[20] Manenti R, Cotelli M, Miniussi C. Successful physiological aging and episodic memory: a brain stimulation study. Behavioural Brain Research 2011;216:153-8.

[21] Rami L, Gironell A, Kulisevsky J, Garcia-Sanchez C, Berthier M, Estevez-Gonzalez A. Effects of repetitive transcranial magnetic stimulation on memory subtypes: a controlled study. Neuropsychologia 2003;41:1877-83.

[22] Rossi S, Cappa SF, Babiloni C, Pasqualetti P, Miniussi C, Carducci F, et al. Prefrontal cortex in long-term memory: an interference approach using magnetic stimulation. Nature Neuroscience 2001;4:948-52.

[23] Sandrini M, Cappa SF, Rossi S, Rossini PM, Miniussi C. The role of prefrontal cortex in verbal episodic memory: rTMS evidence. Journal of Cognitive Neuroscience 2003;15:855-61.

[24] Manenti R, Cotelli M, Robertson IH, Miniussi C. Transcranial brain stimulation studies of episodic memory in young adults, elderly adults and individuals with memory dysfunction: a review. Brain Stimulation 2012;5:103-9.

[25] Davis SW, Dennis NA, Daselaar SM, Fleck MS, Cabeza R. Que PASA? The posterior-anterior shift in aging. Cerebral Cortex 2008;18:1201-9.

[26] Daniele A, Giustolisi L, Silveri MC, Colosimo C, Gainotti G. Evidence for a possible neuroanatomical basis for lexical processing of nouns and verbs. Neuropsychologia 1994;32:1325-41.

[27] Perani D, Cappa SF, Schnur T, Tettamanti M, Collina S, Rosa MM, et al. The neural correlates of verb and noun processing. A PET study. Brain 1999;122(Pt 12):2337-44.

[28] Price CJ, Devlin JT, Moore CJ, Morton C, Laird AR. Meta-analyses of object naming: effect of baseline. Human Brain Mapping 2005;25:70-82.

[29] Barresi BA, Nicholas M, Connor LT, Obler L, Albert ML. Semantic degradation and lexical access in age-related naming failures. Aging, Neuropsychology and Cognition 2000; 7:169-78.

[30] Connor LT, Spiro A, 3rd, Obler LK, Albert ML. Change in object naming ability during adulthood. Journals of Gerontology Series B, Psychological Sciences and Social Sciences 2004;59:P203-9.

[31] Morrison CM, Hirsh KW, Duggan GB. Age of acquisition, ageing, and verb production: normative and experimental data. Quarterly Journal of Experimental Psychology A, Human Experimental Psychology 2003;56:705-30.

[32] Goral M, Spiro 3rd A, Albert M, Obler LK, Connor LT. Change in lexical-retrieval skills in adulthood. The Mental Lexicon 2007:215-38.

[33] Verhaeghen P. Aging and vocabulary scores: a meta-analysis. Psychology and Aging 2003; 18:332-9.

[34] Burke DM, Shafto MA. Aging and language production. Current Directions in Psychological Science 2004;13:21-4.

[35] Shafto MA, Burke DM, Stamatakis EA, Tam PP, Tyler LK. On the tip-of-thetongue: neural correlates of increased word-finding failures in normal aging. Journal of Cognitive Neuroscience 2007;19:2060-70.

[36] Shafto MA, Stamatakis EA, Tam PP, Tyler LK. Word retrieval failures in old age: the relationship between structure and function. Journal of Cognitive Neuroscience 2010;22:1530-40.

[37] Stamatakis EA, Shafto MA, Williams G, Tam P, Tyler LK. White matter changes and word finding failures with increasing age. PLoS ONE 2011;6:e14496.

[38] Berlingeri M, Bottini G, Danelli L, Ferri F, Traficante D, Sacheli L, et al. With time on our side? Task-dependent compensatory processes in graceful aging. Experimental Brain Research 2010;205:307-24.

[39] Berlingeri M, Sacheli L, Danelli L, Ferri F, Traficante D, Basilico S, et al. Neurofunctional and neuromorphological evidence of the lack of compensation in pathological aging. Behavioural Neurology 2010;23:185-7.

[40] Cabeza R, Locantore JK, Anderson ND. Lateralization of prefrontal activity during episodic memory retrieval: evidence for the production-monitoring hypothesis. Journal of Cognitive Neuroscience 2003;15:249-59.

[41] Eyler LT, Sherzai A, Kaup AR, Jeste DV. A review of functional brain imaging correlates of successful cognitive aging. Biological Psychiatry 2011;70:115-22.

[42] Chee MW, Goh JO, Venkatraman V, Tan JC, Gutchess A, Sutton B, et al. Agerelated changes in object processing and contextual binding revealed using fMR adaptation. Journal of Cognitive Neuroscience 2006;18:495-507.

[43] Voss MW, Erickson KI, Chaddock L, Prakash RS, Colcombe SJ, Morris KS, et al. Dedifferentiation in the visual cortex: an fMRI investigation of individual differences in older adults. Brain Research 2008;1244:121-31.

[44] Beason-Held LL, Kraut MA, Resnick SM. Stability of default-mode network activity in the aging brain. Brain Imaging and Behaviour 2009;3:123-31.

[45] Fridriksson J, Morrow KL, Moser D, Baylis GC. Age-related variability in cortical activity during language processing. Journal of Speech, Language, and Hearing Research 2006;49:690-7.
[46] Wierenga CE, Benjamin M, Gopinath K, Perlstein WM, Leonard CM, Rothi LJ, et al. Age-related changes in word retrieval: role of bilateral frontal and subcortical networks. Neurobiology of Aging 2008;29:436-51.

[47] Miniussi C, Ruzzoli M, Walsh V. The mechanism of transcranial magnetic stimulation in cognition. Cortex 2010;46:128-30.

[48] Epstein CM, Sekino M, Yamaguchi K, Kamiya S, Ueno S. Asymmetries of prefrontal cortex in human episodic memory: effects of transcranial magnetic stimulation on learning abstract patterns. Neuroscience Letters 2002;320:5-8.

[49] Kohler S, Paus T, Buckner RL, Milner B. Effects of left inferior prefrontal stimulation on episodic memory formation: a two-stage fMRI-rTMS study. Journal of Cognitive Neuroscience 2004;16:178-88.

[50] Manenti R, Cotelli M, Calabria M, Maioli C, Miniussi C. The role of the dorsolateral prefrontal cortex in retrieval from long-term memory depends on strategies: a repetitive transcranial magnetic stimulation study. Neuroscience 2010;166:501-7.

[51] Manenti R, Tettamanti M, Cotelli M, Miniussi C, Cappa SF. The neural bases of word encoding and retrieval: a fMRI-guided transcranial magnetic stimulation study. Brain Topography 2010;22:318-32.

[52] Rossi S, Pasqualetti P, Zito G, Vecchio F, Cappa SF, Miniussi C, et al. Prefrontal and parietal cortex in human episodic memory: an interference study by repetitive transcranial magnetic stimulation. European Journal of Neuroscience 2006;23:793-800.

[53] Rossi S, Miniussi C, Pasqualetti P, Babiloni C, Rossini PM, Cappa SF. Agerelated functional changes of prefrontal cortex in long-term memory: a repetitive transcranial magnetic stimulation study. Journal of Neuroscience 2004;24:7939-44.

[54] Cappa SF, Sandrini M, Rossini PM, Sosta K, Miniussi C. The role of the left frontal lobe in action naming: rTMS evidence. Neurology 2002;59:720-3.

[55] Cotelli M, Manenti R, Rosini S, Calabria M, Brambilla M, Bisiacchi PS, et al. Action, and object naming in physiological aging: an rTMS study. Frontiers in Aging Neuroscience 2010;2:151.

[56] Cabeza R, Anderson ND, Locantore JK, McIntosh AR. Aging gracefully: compensatory brain activity in high-performing older adults. Neuroimage 2002; $17: 1394-402$.

[57] Folstein MF, Folstein SE, McHugh PR. Mini-mental state. A practical method for grading the cognitive state of patients for the clinician. Journal of Psychiatric Research 1975;12:189-98.

[58] Oldfield RC. The assessment and analysis of handedness: the Edinburgh inventory. Neuropsychologia 1971;9:97-113.

[59] Rossi S, Hallett M, Rossini PM, Pascual-Leone A, Group SoTC. Safety, ethical considerations and application guidelines for the use of transcranical magnetic stimulation in clinical practice and research. A consensus statement from the International Workshop on Present and Future of TMS: safety and ethical guidelines. Clinical Neurophysiology 2009;120(12):2008-39.

[60] Lezak M, Howieson D, Loring DW. Neuropsychological assessment. 4th ed. Oxford: University Press; 2004.

[61] Miceli G, Laudanna A, Burani C, Papasso R. Batteria per l'Analisi dei Deficit Afasici. BADA (Battery for Analysis of Aphasic Deficits). Milano: CEPSAG, Università Cattolica del Sacro Cuore; 1994.

[62] Bates E, Andonova E, D’Amico S, Jacobsen T, Kohnert K, Lu CC, et al. Introducing the CRL International Picture-Naming Project (CRL-IPNP). In: Center for Research in Language Newsletter. La Jolla: University of California San Diego; 200012.

[63] Cabeza R, Dennis NA. Frontal lobes and aging: deterioration and compensation. In: Stuss DT, Knights RT, editors. Principles of frontal lobe function. New York: Oxford University Press; 2012.

[64] Cotelli M, Manenti R, Cappa SF, Geroldi C, Zanetti O, Rossini PM, et al. Effect of transcranial magnetic stimulation on action naming in patients with Alzheimer disease. Archives of Neurology 2006;63:1602-4.

[65] Cotelli M, Manenti R, Cappa SF, Zanetti O, Miniussi C. Transcranial magnetic stimulation improves naming in Alzheimer disease patients at different stages of cognitive decline. European Journal of Neurology 2008;15: 1286-92.

[66] Cotelli M, Calabria M, Manenti R, Rosini S, Zanetti O, Cappa SF, et al. Improved language performance in Alzheimer disease following brain stimulation. Journal of Neurology, Neurosurgery and Psychiatry 2011;82: 794-7.

[67] Cotelli M, Manenti R, Alberici A, Brambilla M, Cosseddu M, Zanetti O, et al. Prefrontal cortex rTMS enhances action naming in progressive non-fluent aphasia. European Journal of Neurology 2012;19(11):1404-12.

[68] Cotelli M, Fertonani A, Miozzo A, Rosini S, Manenti R, Padovani A, et al. Anomia training and brain stimulation in chronic aphasia. Neuropsychological Rehabilitation 2011;21:717-41.

[69] Szaflarski JP, Vannest J, Wu SW, DiFrancesco MW, Banks C, Gilbert DL. Excitatory repetitive transcranial magnetic stimulation induces improvements in chronic post-stroke aphasia. Medical Science Monitor 2011;17:132-9. 